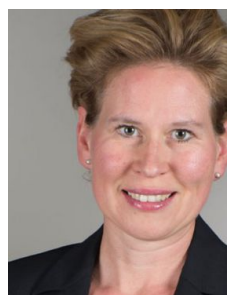

\section{Nicole Bischof}

Dozentin für Organisationsentwicklung (OE) und Kommunikation am Kompetenzzentrum für Leadership und Personalmanagement der OST Ostschweizer Fachhochschule

nicole.bischoft@ost.ch

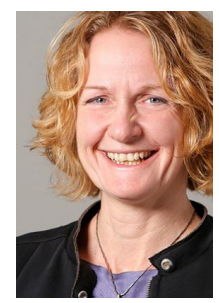

\section{Sibylle Olbert-Bock}

Professorin für Leadership und Personalmanagement der OST Ostschweizer Fachhochschule

sibylle.olbert@ost.ch

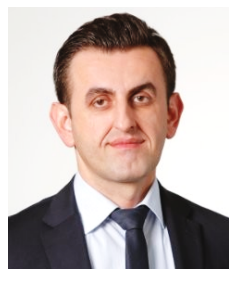

\author{
Abdullah Redzepi \\ Dozent für HR-Management und HR- \\ Development am Kompetenzzentrum \\ für Leadership und Personalmanage- \\ ment der OST Ostschweizer Fach- \\ hochschule \\ abdullah.redzepi@ost.ch
}

\title{
Career Development für Frauen 45+
}

\section{Ein ganzheitlicher Ansatz zur Förderung von Karrierevielfalt in Unternehmen}

Die Karrieren von Frauen entsprechen nach wie vor wenig den traditionellen, männlich geprägten Laufbahnmustern. Von klassischen Mustern abweichendes Handeln wird vonseiten Unternehmen bisher nur wenig unterstützt. Mit wachsendem Berufsalter wird der Preis für Frauen immer höher, den sie für ihr vom männlichen Ideal abweichendes Karriereverhalten in Form eines geringeren objektiven Karriereerfolgs zahlen. Vor dem Hintergrund des wachsenden Anteils an hochqualifizierten Frauen in allen Berufsaltersklassen und dem demografischen Wandel tun Unternehmen gut daran, eine größere Karrierevielfalt zu unterstützen. Eine Veränderung der Förderpraxis in der Gesamtorganisation passt inhaltlich zu den Agilitäts-Bestrebungen zahlreicher Unternehmen. Allerdings stellt es auch einen komplexen Prozess des Wandels dar, der nur gelingt, wenn auf Ebene der Gesamtorganisation mit ihren Strukturen, Kulturen und Instrumenten, auf Ebene von Führung sowie auf Ebene der Individuen aufeinander abgestimmt Maßnahmen in Gang gesetzt werden. Das von uns entwickelte „House of Careers“ und ein zugehöriges Screening unterstützt Unternehmen dabei, dies leisten zu können. 


\section{Weshalb Unternehmen vermehrt auf die Förderung von Frauen 45+ setzen sollten}

Da der demografische Wandel eine Herausforderung für die Deckung des Fachkräftebedarfs darstellt, ist es erstaunlich, dass Frauen 45+ nicht aus Eigeninitiative der Unternehmen in den Fokus ihrer personalpolitischen Ausrichtung rücken. Vielmehr werden nach wie vor Frauen, Ältere und ältere Frauen vorrangig als flexibilitätsorientierte „Manövriermasse" betrachtet, anstelle wie jede andere Arbeitnehmendengruppe anerkannt zu sein. Sie finden sich oft in Beschäftigung wie Teilzeit, Befristungen, Scheinselbständigkeit oder Leiharbeit. Das Stigma der Minderwertigkeit bekommen sie angesichts der fehlenden Integration in interessante Projekte von strategischer Relevanz, systematische Beurteilungen und Beförderungen oft nicht $\operatorname{los}^{1}$.

Das Potenzial von Frauen gilt als noch nicht ausreichend genutzt. Ginge es nach dem Schweizer Bundesamt für Statistik (Scenario 2010 bis 2060) ${ }^{2}$ sollen Frauen in Zukunft höhere Erwerbsquoten erreichen und gelten als besonders interessant zur Lösung von Herausforderungen, die sich in der Schweiz angesichts der demografischen Entwicklung stellen.

Qualifizierte Frauen mit Kindern an sich zu binden, um unter anderem dadurch Geschäftsstrategien umsetzen zu können, ist gegenwärtig eine Herausforderung in vielen Unternehmen. Sie wird sich immer drängender stellen, auch wenn sie aktuell noch extrem zögerlich aufgegriffen wird. Personalentwicklung (PE) im Allgemeinen und die Weiterentwicklung insbesondere der Talente von Frauen 45+ werden in vielen Organisationen auch künftig an Bedeutung gewinnen. Die Unternehmen, die sich frühzeitig für die Unterstützung einer Karrierevielfalt einsetzen, werden einen noch größeren Nutzen erzielen: Männer und junge Menschen generell haben ein wachsendes Interesse an agilen Karrieren. Für Beschäftigungsformen, die dem flexiblen Personaleinsatz dienen, müssen Unternehmen genauso Förderstrategien vorsehen, um ihre Wertigkeit zu steigern und attraktiv zu bleiben.

\section{Entwicklung eines ganzheitlichen Ansatzes zur Förderung von Karrierevielfalt}

Im Rahmen eines 3-jährigen Innovationsprojektes - gefördert vom Eidg. Büro für Gleichstellung - wurden Fördermaßnahmen gezielt für Frauen 45+ entwickelt und in einem Handbuch dargestellt.

Die im Rahmen des Projektes verschiedentlich durchgeführten qualitativen und quantitativen Untersuchungen bei drei Partnerorganisationen ermöglichten die Erfassung der aktuellen Situation mit Blick auf späte Karrieren. Des Weiteren verdeutlichen sie, dass es bei der Frage nach dem Verlauf und der Förderung von weiblichen Karrieren 45+ zahlreiche, verschiedene und ineinander verwobene Handlungsfelder zu berücksichtigen gilt.

In der zweiten Phase des Projektes folgten qualitative Interviews mit Frauen 45+ mit deutlichem Karriereumbruch. Aus dieser Untersuchung konnten Erkenntnisse im 
Hinblick auf bestehende Förder- und Hinderungsmuster für weibliche Karrieren 45+ eruiert sowie eine Vielzahl an Stereotypen aufgedeckt werden. Insgesamt wurden Fragen hinsichtlich der Erwartungen, Erfahrungen und Anforderungen sowohl vom Einzelnen an die Organisation als auch von der Unternehmensseite an Personen 45+ adressiert.

Eines der zentralen Erkenntnisse dieser Untersuchungen ist, dass es auf Dauer nichts nützt, wenn Organisationen zur Stärkung der Situation der Frauen 45+ lediglich einzelne operative Häppchen anbieten. Zwar sind sie ein wichtiger Startpunkt, aber um eine echte Erneuerung der Förderpolitik zu erreichen und eine Karrierevielfalt in Organisationen zu ermöglichen, sollte ein Gesamtkonzept aufgestellt werden ${ }^{3}$.

Solch ein ganzheitlich ausgerichtetes Konzept zur spezifischen Weiterentwicklung sowohl der Gesamtorganisation als auch des Einzelnen wurde im Rahmen dieses Projektes entwickelt - das nachfolgend vorgestellte Modell „House of Careers“ (siehe Abb. 1). Dieses in der Praxis erprobte Konzept greift neben der individuellen Perspektive, die Perspektive der Führung und Gesamtorganisationen auf. Genutzt wurden wissenschaftliche Vorarbeiten ${ }^{4}$ und Erkenntnisse, die im Verlauf des Projektes entstanden sind. Mit dem im Modell verankerten Ansatz können Organisationen eine Basis für die bedarfsorientierte Auswahl von PE-Maßnahmen zur Gestaltung und Förderung von Frauen-Karrieren $45+$ schaffen.

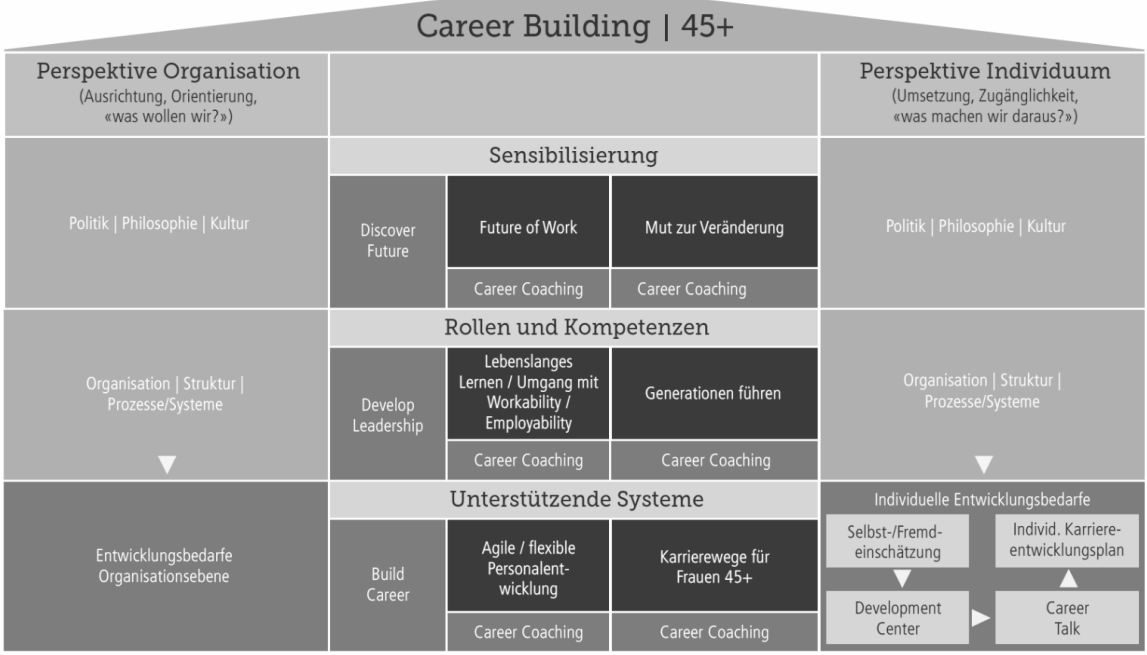

Abbildung 1: House of Careers mit PE-Maßnahmen auf individueller und organisationaler Ebene. ${ }^{5}$ 
Eines der wesentlichen Elemente des Ansatzes ist es, dass eine stärkere Ausdifferenzierung von Karrieren angesichts der aktuellen Lebenssituation und erworbener Expertise vorgenommen wird. Entlang der Themen- und Maßnahmenfelder können Organisationen aus verschiedenen relevanten Maßnahmen auswählen und sich fortwährend weiterentwickeln. Durch konsequente Sensibilisierung, eine gezielte Klärung von Rollen und Kompetenzen sowie der Schaffung von unterstützenden Systemen und Mechanismen können sich Organisation in Karriereentwicklungsfragen nachhaltig ausrichten. Neben einer Anpassung der Förderpolitik, können auch die Förderstrategie und konkrete HR-Instrumente angepasst werden. Nicht nur auf organisationaler Ebene, sondern auch auf Führungs- und individueller Ebene lassen sich bedarfsspezifisch Personalentwicklungsmaßnahmen neukonzipieren.

Damit Organisationen gezielt ihren Handlungsbedarf in puncto Förderung von Frauen 45+ analysieren können, erfolgte die Entwicklung eines Screenings als Instrument zur Organisationsanalyse (siehe Abb. 2) ${ }^{5}$.

\section{Screening für Frauenkarrieren 45+: Instrument zur Organisationsanalyse}

Organisationen, die sich ein umfassendes, analysegestütztes Bild über ihre generelle und auf Frauen 45+ fokussierte Ausrichtung ihrer Personalentwicklung und Karriereförderung verschaffen möchten, können das mithilfe des in Zusammenarbeit mit verschiedenen Praxispartnern entwickelten Screenings tun. Die Besonderheit des Screenings liegt darin, dass Unternehmen Erfolgsfaktoren sowie Problembereiche der operativen Umsetzung erkennen können, um diese in der Folge gezielt anzugehen und die Ausrichtung ihrer Personalentwicklung und Karriereförderung nachhaltig auszurichten. Das entwickelte Screening deckt unterschiedliche Themenbereiche ab und unterscheidet vier Elemente, mit denen eine Status-Quo-Validierung vorgenommen werden kann:
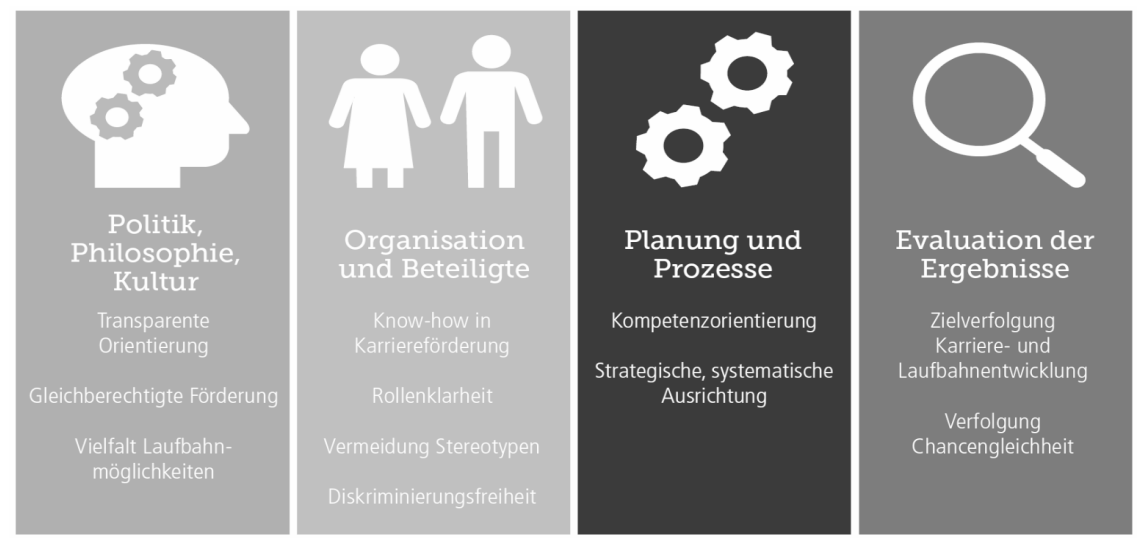

Abbildung 2: Themenfelder des Screenings. ${ }^{5}$ 
Ergänzend werden Organisationen nach Wünschen zu konkreten Maßnahmen befragt. Die Inhalte orientieren sich an Erfolgsfaktoren und Herausforderungen der nachhaltigen Personalentwicklung sowie Karriereförderung generell und von Frauen 45+ in Unternehmen. Konkret sieht das Screening eine Operationalisierung der nachfolgend beschriebenen Aspekte vor:

\begin{tabular}{|c|c|}
\hline Aspekt & Operationalisierung/Beschreibung \\
\hline $\begin{array}{l}\text { Transparente Orientie- } \\
\text { rung }\end{array}$ & $\begin{array}{l}\text { Erfolgreiche Förderung setzt ein hohes Mass an Selbstverpflichtung voraus, die sich } \\
\text { durch Transparenz einfordern und Unterlegung mit finanziellen Mitteln umsetzen } \\
\text { lässt. }\end{array}$ \\
\hline $\begin{array}{l}\text { Vielfalt an Laufbahn- und } \\
\text { Karrieremöglichkeiten }\end{array}$ & $\begin{array}{l}\text { Die Entwicklung von Mitarbeitenden folgt in den jeweiligen Organisationen unter- } \\
\text { schiedlichen Mustern und Mitarbeitende folgen unterschiedliche Wege. Förderung } \\
\text { sollte Vielfalt unterstützen. }\end{array}$ \\
\hline $\begin{array}{l}\text { Gleichberechtigte Förde- } \\
\text { rung }\end{array}$ & $\begin{array}{l}\text { Wendet sich ausdrücklich der Vermeidung von struktureller Diskriminierung zu. Die } \\
\text { unterschiedliche Anerkennung von Stationen in Frauen- und Männerdomänen oder } \\
\text { typisch weiblicher Karrieremuster muss erkannt und ihr muss aktiv begegnet werden. }\end{array}$ \\
\hline $\begin{array}{l}\text { Orientierung an der Stra- } \\
\text { tegie und Personalpla- } \\
\text { nung }\end{array}$ & $\begin{array}{l}\text { Die prognostische Planung des künftigen Personalbestandes in quantitativer und } \\
\text { grundlegender qualitativer Hinsicht, sowie die Gewährleistung der Besetzung von } \\
\text { Schlüsselfunktionen ist ein wesentliches Element strategischen Handelns und des } \\
\text { Vermeidens von Personalrisiken. }\end{array}$ \\
\hline Kompetenzorientierung & $\begin{array}{l}\text { Stellenbesetzungen sollten sich an den Anforderungen orientieren und es gilt Biases } \\
\text { aus unbewussten Präferenzen, etwa entlang eines gedachten «Prototyps» von Füh- } \\
\text { rung zu vermeiden, aus denen einerseits Vorurteile und Benachteiligungen für den } \\
\text { Einzelnen sowie Nachteile für eine optimale Stellenbesetzung für das Unternehmen/ } \\
\text { die Organisation entstehen können. }\end{array}$ \\
\hline Rollenklarheit & $\begin{array}{l}\text { Angesichts zunehmender Einforderung von Selbstverantwortung für Entwicklung und } \\
\text { Förderung, die oft «klassische» Karrieren ablöst sind Erwartungen an die Beteiligten } \\
\text { zu klären und offen zu legen, sowie Missverständnisse zu vermeiden. }\end{array}$ \\
\hline $\begin{array}{l}\text { Know-How in der Karrie- } \\
\text { reförderung }\end{array}$ & $\begin{array}{l}\text { Gute Prozesse und Praktiken nützen nur dann, wenn sie gelebt werden. Vorausset- } \\
\text { zung ist, dass Wissen über die Ziele und die korrekte Umsetzung von Praktiken be- } \\
\text { steht sowie die erforderlichen Ressourcen zu ihrer Umsetzung zur Verfügung stehen. }\end{array}$ \\
\hline $\begin{array}{l}\text { Diskriminierungsfreiheit } \\
\text { und Vermeidung von } \\
\text { Stereotypen }\end{array}$ & $\begin{array}{l}\text { Bezogen auf Alter und Geschlecht bestehen zahlreiche Formen der Diskriminierung, } \\
\text { die auf nicht offen gelegten Stereotypisieren basieren und in Selbstdiskriminierung } \\
\text { münden können. Hierzu zählen etwa ausdrückliche Bevorzugungen jüngerer Bewer- } \\
\text { ber aufgrund beispielsweise der Unflexibilität Älterer, die darin münden können, dass } \\
\text { Ältere für sie Weiterentwicklung gar nicht mehr in Erwägung ziehen. Es gilt sie auf- } \\
\text { zudecken und einen sie begünstigenden Diskurs zu unterbinden. }\end{array}$ \\
\hline
\end{tabular}

Tabelle 1: Das Screening sieht eine Operationalisierung der beschriebenen Aspekte vor.

\section{Personalentwicklungsmaßnahmen für das „House of Careers“}

Für das „House of Careers" werden je nach Unternehmensbedarf Personalentwicklungsmaßnahmen in Form von halbtägigen Quick-Wins ausgearbeitet, die auf die Besonderheiten in Zusammenhang der Förderung von Frauenkarrieren 45+ reagieren. 
Beispielhafte Quick-Wins, die integraler Bestandteil des House of Careers sind, sind die Unterstützung selbstgesteuerter Karrieren oder die Sensibilisierung für Alters- und Genderstereotypen. Einige der Maßnahmen wurden in der Praxis getestet. Ein Beispiel ist der nachfolgend zusammengefasste Baustein «Generationen führen und Potentiale fördern».

\section{i Workshop: Generationen führen und Potenziale fördern}

Unter dem Dach der Gebrüder Weiss Holding AG mit Sitz in Lauterach, Vorarlberg, fasst das Unternehmen neben den Hauptgeschäftsbereichen Landtransporte, Luft- und Seefracht sowie Logistik auch eine Reihe von leistungsfähigen Speziallösungen und Tochterunternehmen zusammen (www.gw-world.com). Die Personalpolitik des Unternehmens setzt auf die Förderung und Bindung ihrer Mitarbeitenden. In diesem Zuge konnten wir ein Training zum Thema 'Generationen führen' im Unternehmen umsetzen und in altersdiversen Teams Impulse für eine erfolgreiche Zusammenarbeit setzen.

Folgende Elemente waren Bestandteile des Trainings:

- Quick-Win-Seminar mit Teamleitdenden aus verschiedenen Regionen

- Situationsanalyse: Visualisierung der Ist-Situation

- Erkenntnisgewinn: dynamische Rollenmodelle und State-of-the-art der Generationsforschung

- Zielbildformulierung: Visualisierung der Ziel-Situation

- Massnahmenplanung: mein weiteres Vorgehen als Führungskraft

- Key-Take-Aways: Diversität fordert heraus und fördert dabei.

Es wurden verschiedene sozialpsychologische Modelle vorgestellt, die bei den Teilnehmenden zu grossem Erkenntnisgewinn geführt haben, so zum Beispiel, dass die 'Omega-Position' im Team sehr wertvoll ist und durch kritisches Feedback das Team in der eigenen Entwicklung voranbringt. «Jetzt sehe ich den Wert unseres ältesten Teammitgliedes, der immer alles kritisch hinterfragt!» (Teamleitende).

\section{Fazit - Handlungsempfehlungen: Was lässt sich aus diesen Erkenntnissen für die Praxis ableiten?}

Die Ergebnisse unserer Untersuchung zeigen, dass Unternehmen handeln müssen. Für die Zielgruppe «ältere Frauen» gibt es wenig bis kaum Förderung in Unternehmen.

Bedenkt man, dass diese Personengruppe eine langjährige Zugehörigkeit zum Unternehmen und einen hohen Expertenstatus aufweist, ist es umso wichtiger, diese Zielgruppe an das Unternehmen zu binden und sie nicht viele Jahre vor der Pensionierung zu verlieren. Das Unternehmen zu einem Zeitpunkt zu verlassen, an dem Frauen $45+$ in eine Schlüsselposition im Unternehmen einnehmen, geschieht meist aufgrund fehlender Sinnhaftigkeit und mangelndem Gestaltungsspielraum ${ }^{6}$. Während sich Jüngere teilweise gut durch berufliche Aufstiegsmöglichkeiten oder monetäre Anreize motivieren 
lassen, streben Ältere eher nach Sinnhaftigkeit ihrer Tätigkeit und Freude bei der Arbeit, also intrinsische Motivatoren. Für die Wirtschaft bedeutet das, dass Ältere im Unternehmen gehalten werden können, wenn man ihnen Gelegenheit zur Gestaltung, Verantwortungsübernahme ${ }^{7}$ und Sinnhaftigkeit bietet bspw. durch die Weitergabe ihres Wissens oder durch die Tätigkeit als Mentorin.

Eine zweite Erkenntnis betrifft die Sensibilisierung für das Thema ,Unterbindung von Diskriminierung'. Auch das unterschwellige Pflegen bzw. Nicht-Bekämpfen von Altersund Genderstereotypen kann als eine Art der Diskriminierung betrachtet werden ${ }^{8}$. Wie werden bspw. Stellenausschreibungen formuliert oder Unternehmenswebseiten bebildert? Sehr selten sieht man hier alters- und genderdiverse Teams. Unsere Empfehlung geht sogar einen Schritt weiter und empfiehlt Unternehmen die aktive Nutzung von Alters- und Genderdiversität für das Employer Branding. Neben der unternehmensinternen und -externen Kommunikation, sollte hier auch die Durchgängigkeit von Personalentwicklungsmaßnahmen gesehen werden, also auch die Förderung von Älteren.

Die dritte Erkenntnis, die wir aus unserer Studie ableiten können, betrifft die Personalpolitik und das Risikomanagement des Unternehmens. Während sich viele Risikofaktoren gut in Zahlen darstellen lassen, wie z.B. das Wegbrechen von Marktanteilen, Produktionsausfall aus technischen Gründen, große Opportunitätskosten bei Investitionen in Technisierung, kann die 'innere Kündigung' von Schlüsselpersonen mit langjähriger Unternehmenserfahrung ${ }^{9}$ in der Regel nicht monetär beziffert werden. Dieses große unternehmerische Risiko fällt häufig erst dann auf, wenn es zu spät ist. Viele Unternehmer sind sich dessen nicht bewusst reagieren nicht aktiv darauf. Mit einer transparenten und klar kommunizierten Personalpolitik ${ }^{10}$, die auch - oder vor allem - späte, weibliche Karrieren fördert, kann aktiv Risikomanagement betrieben und dieses Unternehmensrisiko deutlich reduziert werden.

\section{Literatur}

1 Jensen. P. H. (2020). Nationale Politiken zur Verlängerung der Lebensarbeitszeit und Auswirkungen auf soziale Ungleichheiten. Naegele, G. \& Hess, M. (Hrsg.). Alte und neue soziale Ungleichheiten bei Berufsaufgabe und Rentenübergang.

2 Bundesamt für Statistik (2010). Szenarien zur Bevölkerungsentwicklung der Schweiz 2010-2060. Bern: Schweizerische Eidgenossenschaft.

3 Olbert-Bock, S., Redzepi, A., Cloots, A. \& Marting, R. (2015). Strategische Personalentwicklung in KMU-Netzwerken - Entwicklung von unternehmensübergreifenden Lösungen des Talentmanagements in der IT. St. Gallen: FHS St.Gallen.

4 Redzepi, A., Cloots, A. \& Olbert-Bock, S. (2016) Personalentwicklung als unternehmerische Herausforderung. In: KMU-Magazin, 4/16, S. 84-88.

Olbert-Bock, S./ Redzepi, A. (2018). Digitalisierung und ihre Bedeutung für Arbeit und Personalpolitik. In: Köhler, C./ Olbert-Bock, S./ Strittmatter, M. (2018): Grünbuch DIGITALE AGENDA BODENSEE - Eine Bestandsaufnahme zum Potential der Digitalisierung innerhalb KMU in der Bodenseeregion. 22-33. 
5 Olbert-Bock, S., Redzepi, A., Bischof, N. (2020). Screening für Frauenkarrieren 45+: Instrument zur Organisationsanalyse. in: Olbert-Bock, S., Cloots, A., Graf, U.(Hrsg.): Innovation im HR und Career Development von Frauen $45+$. Unternehmensprozesse und Fördermassnahmen. FHS St. Gallen.

6 Bischof, N. \& Olbert-Bock, S. (2020). Karrieren von Frauen, Älteren und älteren Frauen. in: Olbert-Bock, S., Cloots, A., Graf, U.(Hrsg.): Innovation im HR und Career Development von Frauen 45+. Unternehmensprozesse und Fördermassnahmen. FHS St. Gallen.

7 Bischof, N., Olbert-Bock, S., Redzepi, A. (2020). Späte Frauenkarrieren mit Transition. in: Olbert-Bock, S., Cloots, A., Graf, U.(Hrsg.): Innovation im HR und Career Development von Frauen 45+. Unternehmensprozesse und Fördermassnahmen. FHS St. Gallen.

8 Buengeler, C., Leryo, H., Stobbeleri, K. de (2018). How leaders shape the impact of HR's diversity practices on employee inclusion. Human Resource Management Review 28, 289-303.

9 Darouei, M. \& Pluut, H. (2018). The paradox of being on the class cliff: why do women accept risky leadership positions? Career Development International 23. 397-426.

10 Schröder-Kunz, S. (2018). Generationen (gut) führen. Altersgerechte Arbeitsgestaltung für alle Mitarbeitergenerationen. Springer-Gabler. https://doi.org/10.1007/978-3-658-24097-4.

\section{Information zu den Autorlnnen}

Dr. oec. HSG Nicole Bischof ist Dozentin für Organisationsentwicklung (OE) und Kommunikation am Kompetenzzentrum für Leadership und Personalmanagement der OST Ostschweizer Fachhochschule. Sie beforscht nebst OE auch Personal- und Teamentwicklungsthemen, wie Formen der Zusammenarbeit, Self-Leadership und Life-Design. Aktuell baut sie ein Mentoringprogramm für Frauen auf, um diese auf ein Mandat in Aufsichtsräten vorzubereiten.

Prof. Dr. rer. pol. Sibylle Olbert-Bock, Professorin für Leadership und Personalmanagement der OST Ostschweizer Fachhochschule hat umfassende Leitungserfahrung des Kompetenzzentrums und seiner Forschung, Lehre und Dienstleistung. Eigene Schwerpunkte liegen in Fragestellungen der Modernisierung von Personalführung \& Digitalisierung, Diversity \& Förderung von Chancengleichheit, nachhaltiger Unternehmensentwicklung \& HR Governance.

Abdullah Redzepi, MSc in Business Administration, ist Dozent für HR-Management und HR-Development am Kompetenzzentrum für Leadership und Personalmanagement der OST Ostschweizer Fachhochschule. Seine Forschungs- und Beratungsschwerpunkte liegen insbesondere in der unternehmensspezifischen Ausrichtung von Kompetenz-Modellen, in der Implementierung nachhaltiger Kompetenzmanagementlösungen und dazu passender Führungstools sowie bedarfsspezifischer PE-Programme für Fach- und Führungskräfte. 